\title{
Analysis of the spatial distribution and clinical features of prostate cancer in transperineal prostate biopsy
}

\section{Chen Jia-Jun}

Zhejiang University School of Medicine

Zhu Zai-Sheng ( $\square$ zaisheng_zhu@163.com )

Jinhua Municipal Central Hospital https://orcid.org/0000-0003-4614-9479

\section{Zhu Yi-Yi}

Zhejiang University School of Medicine

\section{Zhou Yi-Bo}

Jinhua Municipal Central Hospital

Shi Hong-Qi

Jinhua Municipal Central Hospital

\section{Research article}

Keywords: Prostate cancer, Prostate cancer risk, Puncture indication, Spatial distribution, Transperineal prostate biopsy

Posted Date: July 26th, 2019

DOI: https://doi.org/10.21203/rs.2.11905/v1

License: () (1) This work is licensed under a Creative Commons Attribution 4.0 International License. Read Full License 


\section{Abstract}

Background Recently, most studies on the spatial distribution of the prostate cancer are based on the samples confirmed by transrectal prostate biopsy (TRBx), which could distinguish the distribution of cancer lesions between the apex, middle and basal parts of the prostate, but the distinction between lesions in the left and right sides of prostate cancer and the transitional and peripheral bands remains to be considered. Further, there has been little research on the specific proportion of cancer in prostate biopsy tissue. The current study aimed to analyze the clinical characteristics, diagnostic efficacy of relevant indicators, and reveal the spatial distribution of prostate cancer in transperineal prostate biopsy (TPBx). Methods A total of 810 patients underwent TPUS-guided 10-core prostate biopsy in our hospital from Oct. 2016-to Feb. 2019, participants' clinical data and the diagnostic yield of the cores were recorded and retrospectively analyzed as a cross-sectional study. Results Age, total prostate specific antigen (t-PSA), prostate volume (PV), prostatic inflammation, dysuria, hematuria, asymptomatic and MRI were independent factors in prostate cancer (Pca) patients compared with non-Pca patients $(P<0.05)$. The cut-off points for age, t-PSA, free prostate specific antigen (f-PSA), PSA density (PSAD), free/total prostate specific antigen ( $f / t P S A$ ) and PV were 73years old, $15.43 \mathrm{ng} / \mathrm{ml}, 4.545 \mathrm{ng} / \mathrm{ml}, 0.475 \mathrm{ng} / \mathrm{ml}{ }^{*} \mathrm{~cm} 3,0.123$ and $41.45 \mathrm{ml}$, respectively. The PRPN of left peripheral zone (LPZ) prostate tumor was elevated regardless of the Gleason score. However, the PRPN of left transitional zone (LTZ) was lower than LPZ and similar to right peripheral zone (RZ), but PRCF and CFVR were significantly higher, especially in tumors with higher Gleason score $(\geq 8)$. Conclusions For Chinese, the $t-P S A$ standard and the PSAD standard in the puncture indication should be increased, while the $\mathrm{f} / \mathrm{t}$ PSA standard should be reduced. At the same time, multi-factor assessment is needed to determine whether patients need a prostate biopsy or not. The spatial distribution of prostate cancer is asymmetrical, with more cancer lesion on the left than on the right. The PRPN of LPZ is relatively higher. LTZ has higher PRCF, and most of them were large lesions with high Gleason score $(\geq 8)$.

\section{Background}

Prostate cancer is the most common malignant tumor of male reproductive system. In 2018 US, the incidence of Pca in male malignant tumors ranked first and the mortality rate ranked second [1]. In recent years, the incidence of $\mathrm{Pca}$ in China has increased rapidly due to changes in lifestyle, aging population and the gradual promotion of prostate-specific antigen screening [2-3]. Since the invention of transrectal ultrasound-guided transrectal prostate biopsy (TRBx) by Hodge et al. in 1989 [4], prostate biopsy became the gold standard in the diagnosis of Pca. However, for the selection of prostate biopsy indications, improve the positive rate of puncture while avoiding over-diagnosis, and the definition of the spatial distribution of tumor in the prostate are still hot issues in the biopsy of Pca [5].

This study discussed the risk of Pca, the diagnostic efficacy of various laboratory indicators, and the spatial distribution characteristics of Pca tissues by analyzing the clinical data of patients undergoing TPBX.

\section{Methods}

\subsection{Enrollment}


There was a total of 810 patients underwent TPBx in our hospital between Oct. 2016 and Feb. 2019, among whom 758 patients met the puncture criteria. (Puncture standard: $\nabla$.Anomalous nodules of the prostate were detected by digital rectal examination, ultrasound, CT or MRI. $\nabla$. T-PSA > $10 \mathrm{ng} / \mathrm{ml} ; \nabla .10 \mathrm{ng} / \mathrm{ml}>\mathrm{t}-\mathrm{PSA}>$ $4 \mathrm{ng} / \mathrm{ml}, \mathrm{PSAD} \geq 0.15 \mathrm{ng} / \mathrm{ml} / \mathrm{cm}^{3}$ or free/total prostate specific antigen (f/t-PSA) $\leq 0.16$. (2016 consensus of Chinese experts on prostate puncture [5])). There were 271 cases of Pca diagnosed by TPBx, and the positive rate of TPBx was $35.8 \%$. In 74 cases, the biopsy only recorded positive or negative results due to a lack of specific proportion of prostate cancer tissue. Among these 271 patients with Pca, 120 of them were treated with radical prostatectomy (RP) with adjuvant Hormonal Therapy (AHT), and they had postoperative pathological analysis (Fig. 1).

\subsection{Puncture Method}

This study was approved by the Ethics Review committee of Jinhua Central Hospital, and all patients signed the informed consent before the puncture. Professor Yi-Bo Zhou performed TPBx on all patients (with more than 10 years of experience in prostate biopsy). The past history of all patients was recorded and auxiliary investigations such as PSA and MRI were done. $10+X$ core prostate biopsy (Fig. 2) (On transverse section, for 10-core TPBx, eight cores were sampled from the peripheral zone (PZ) and the other two were from the transitional zone (TZ). When the prostate is larger than $50 \mathrm{~g}$, four cores were added to the PZ of the prostate apex. The length of each needle puncture tissue varies $0.5-1.5 \mathrm{~cm}$.). Pathological analysis of all prostate specimens was performed by Professor Hong-Qi Shi, who also collected the puncture pathology data of patients who performed RP.

\subsection{Statistical methods}

The measurement data were tested by K-S normality. Univariate analysis was carried out to compare the differences of each factor in the diagnosis of prostate cancer: Independent-sample T-test was performed on the measurement of data conforming to the normal distribution, and the Wilcoxon signed rank sum test of two independent samples was selected for the other measurement of data. The chi-square test was selected for the differences among the classification variables. Significant influencing factors were screened out and analyzed by binary logistic regression to investigate the specific influence of variables on prostate cancer. The ROC curve was used to measure the measurement data which had a significant effect on the diagnosis of prostate cancer, and the cut-off point was found out according to Youden index. The puncture data of prostate cancer patients were grouped according to different Gleason scores and different puncture sites, and chi-square test was performed to analyze whether there was any difference in the PRPN, the CFVR and the PRCF. Paired-samples t-test was used to compare the difference between puncture pathological data and postoperative pathological data of patients with RP. (The PRPN = The number of positive puncture needles / The total number of puncture needles; The CFVR = The volume of Pca lesion / The volume of tissue obtained in positive puncture needles; The PRCF = The volume of Pca lesion / The total volume of prostate tissue obtained)

\section{Results}

\subsection{Comparison of clinical manifestations and auxiliary examination of 758 puncture patients (Table 1)}


There was a large amount of bias in the data of patients' digital rectal examination, only 3 patients who obviously felt nodules, so digital rectal examination was not included in the multi-factor analysis. All patients had no previous puncture history and no family history of Pca. The medium age of Pca patients was 74 (range 51 97) years old, while non-Pca patients was 67 (range $35 \sim 90$ ) years old. The medium tPSA of Pca patients was 24.36 (range $0.57 \sim 1000 \mathrm{ng} / \mathrm{L}$ ), while non-Pca patients was 10.83 (range $0.25 \sim$ $146.46 \mathrm{ng} / \mathrm{L}$ ). The medium PV of Pca patients was 35.3 (range 12.5囚151.9 ml), while non-Pca patients was 41.9 (range 10.9ه144.0 ml). There was no significant difference in BMI between the Pca group and the nonPca Group $(P=0.385)$, and there were significant differences in age, t-PSA, f-PSA, f/t PSA, PV and PSAD $(P<$ 0.001). Prostate cancer is associated with prostatitis, dysuria, hematuria, asymptomatic, hypertension, calcification and MRI.

$\mathrm{PV}$ is a protective factor for prostate cancer ( $\mathrm{OR}=0.986,95 \% \mathrm{Cl}: 0.975$ to $0.997, \mathrm{P}=0.013)$, the risk of prostate cancer was increased in patients with non-prostatitis $(\mathrm{OR}=5.793,95 \% \mathrm{Cl}: 3.285$ to $10.217, \mathrm{P}<$ 0.001 ), non-dysuria $(\mathrm{OR}=10.555,95 \% \mathrm{Cl}: 4.015$ to $27.751, \mathrm{P}<0.001)$, and non-hematuria $(\mathrm{OR}=4.708,95 \%$ Cl:1.523 to $14.555, P=0.007)$.

\subsection{Puncture Results}

Results of 181 patients (gleason score $8 \sim 10)$ : The PRPN of LZ was higher than that of $R Z(X 2=2.919, P=$ $0.088<0.1)$. The PRPN of LPZ was higher than that of RTZ $(X 2=4.393, p=0.036)$, and there was no difference between the other parts. The CFVR of LTZ was higher than that of LPZ, RPZ and RTZ $(X 2=3.075$, $P=0.079<0.1 ; \chi 2=6.886, P=0.009 ; \chi 2=4.462, P=0.035)$, and there was no difference between the other

parts. The PRCF of $L Z$ was higher than that of RZ $(X 2=12.426, P<0.001)$. The CFVR of $L P Z$ was higher than that of RPZ and RTZ $(X 2=6.942, P=0.008 \square X 2=3.368, P=0.066<0.1)$, the CFVR of LTZ was higher than that of RPZ and RTZ ( $X 2=9.940, P=0.002 \square X 2=6.246, P=0.012)$, and there was no difference between the other parts(Table 6).

There was a statistical difference between puncture pathological data and postoperative pathological data of 120 patients with RP. The mean Gleason score of puncture pathology and surgical pathology was 7.1167 and 6.8417, respectively, and there was a significant correlation between them $(P<0.001)$. The Gleason score of surgical pathology was 0.275 higher than that of puncture pathology $(T=4.176, P<0.001)$.

\section{Discussion}

Transrectal ultrasound-guided TRBx is the main method to diagnose prostate cancer without surgery $[6,7]$. However, false negative rate (FNR) is higher in transrectal puncture, which is reported to be up to $15 \%$ $31 \%$ $[8,9,10]$. It was reported that TPBx could significantly improve the detection rate of tumors in the anterior half and apex of the prostate compared with TRBx $[11,12,13]$, and can accurately assess the PV, at the same time, it has the advantage of safety and low infection rate, transperineal biopsy is becoming more and more popular in prostate biopsy $[6,14]$. However, better selection of prostate biopsy indications, improvement of the positive rate of puncture of the prostate are still hot issues [5]. 
The positive rate of TPBx in this study was 35.8\%, slightly higher than the rate (34.5\%) reported by Pepe $\mathrm{P}$ et al [15], but generally lower than that reported by foreign literatures [16, 17]. This difference may be mainly caused by racial difference, because the incidence of Pca in European and American is much higher than that in China, and the differences caused by such factors as inconsistent pathological diagnostic criteria cannot be excluded.

The cut-off points for age, t-PSA, f-PSA, PSAD, f/t PSA and PV were 73 years old, $15.43 \mathrm{ng} / \mathrm{ml}, 4.545 \mathrm{ng} / \mathrm{ml}$, $0.475 \mathrm{ng} / \mathrm{ml}^{*} \mathrm{~cm} 3,0.123$ and $41.45 \mathrm{ml}$, respectively. Compared with the current puncture guidelines [5], t-PSA and PSAD are higher, while f/t PSA is lower, which may be related to the fact that the enrolled patients have been screened by the guidelines. Compared with the Pca patients in the United States reported in the literature $[18,19]$, the results of this study showed that Pca in Chinese had the characteristics of older age, higher t-PSA and smaller PV. In terms of clinical manifestations, this study found that the positive rate of TPBx in symptomless patients was higher, and lower in patients with prostatitis, dysuria and hematuria. This may be related to the fact that most of the patients received TPBx only because of abnormal prostate screening, and most of the patients' dysuria and hematuria and other symptoms may be caused by diseases such as benign prostatic hyperplasia.

Compared with TRBx, TPBx significantly increased the tissue acquisition of TZ in the prostate, and also increased the tissue acquisition of PZ to a certain extent. However, TPBx is prone to omission in the detection of basal tumors. Although the incidence of prostate cancer is not high in the basal region [20,21], the puncture of the basal region should be paid more attention in TPBx, especially for patients with imaging space occupying near the basal region. As it is reported that in the $P Z$, especially in the apex of prostate, is the most common site of Pca. Breslow et al. [20] analyzed the results of 1327 autopsies in seven centers around the world and found 350 cases of latent Pca, which mainly appeared in the PZ, most of which were $5 \sim 15 \mathrm{~mm}$ from the apex of prostate, and the tumor incidence was the same in the anterior and posterior regions. The results of 271 Pca patients in this study suggested that the PRPN of PZ was higher than that of TZ, which was consistent with the literature. However, further comparison revealed that the PRPN of LZ was higher, especially the PRPN of LPZ was significantly higher than that of the rest; When CFVR was compared, it was found that the CFVR of LTZ was significantly larger than that of the other regions; When comparing PRCF, we found that the PRCF of LPZ and LTZ were significantly higher than that of RPZ or RTZ, and there was no significant statistical difference between LPZ and LTZ, RPZ and RTZ. This result may be caused by the existence of a large number of small cancer foci in the LPZ, a small number of large cancer foci in the LTZ, and a small number of small cancer foci in the RPZ and the RTZ. We draw the conclusion that: Increasing the puncture in the LPZ may improve the detection rate of Pca. Increasing the puncture in the LTZ may allow more accurate staging of patients to guide subsequent treatment. Although the positive rate of RZ puncture is lower than that of LZ, the puncture in the RZ should not be neglected.

Gleason scoring system has become the standard pathological grading system for Pca in WHO since 1993 [22], it has become the most important indicator for evaluating the invasion of tumors and determining the prognosis of patients [23], and the accuracy of predicting the progression of localized Pca is 30\% 69\% [24]. As it was reported in the study results of the largest international prostate puncture collaboration group that the proportion of high-level Pca (Gleason score 7 10) in clinical cohort in European and American countries 
is $30 \%$ and $51 \%$ respectively. In our study, the proportion was $62 \%$, significantly higher than that reported in the literature. This may be related to racial difference, or it may be caused by different pathological evaluation criteria (the new grading system of 2015 version [27] was adopted in our study). When cancer lesions were further divided into different Gleason scores. There was no difference in CFVR and PRCF between different areas (Gleason score 6-7). The PRPN of LPZ was higher than that of other regions, and the CFVR of LTZ was significantly higher than that of other regions (Gleason score 8-10). This result further indicates that the high PRCF of LZ may be related to the high Gleason score of Pca lesions in the LZ. We compared the biopsy pathology and postoperative pathology of 120 patients with RP after puncture, and found that the Gleason score of postoperative pathological was 0.275 points higher than that of puncture pathology, which may be related to the fact that more tissues can be obtained from RP for more accurate pathological assessment.

Limitations: $\mathbb{\|}$.This paper is a retrospective study with a small sample size and possible bias in data. Therefore, it is necessary to further expand the sample size. . It was a single-center study and lacked a contrast in the study of the spatial distribution of Pca by TRBX. 囚.Digital rectal examination was not included

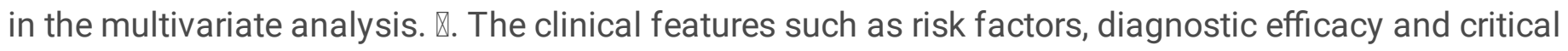
values of t-PSA in gray area ( $4 \sim 10 \mathrm{ng} / \mathrm{ml}, 2016$ consensus of Chinese experts on prostate puncture [5]) were not analyzed. $\$. Patients in our study group have been screened by puncture guidelines, and the cut-off point of t-PSA obtained may be relatively high, the obtained critical value of $f / t$ PSA and PSAD is only the

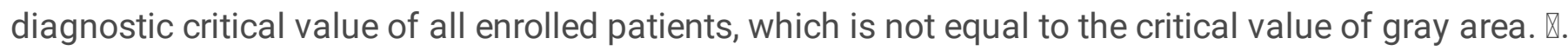
Postoperative pathology of RP patients was recorded only by the highest Gleason score, and the distribution of cancer lesions was not recorded in different regions and analyzed.

\section{Conclusion}

To sum up, based on the data of 758 TPBx patients in our hospital, we analyzed the clinical characteristics, diagnostic efficacy of relevant indicators, and spatial distribution of Pca in Chinese. We found that for Chinese, the t-PSA standard and the PSAD standard in the puncture indication should be increased, while the $\mathrm{f} / \mathrm{t}$ PSA standard should be reduced. At the same time, multi-factor assessment is needed to determine whether patients need a prostate biopsy or not. The spatial distribution of prostate cancer is asymmetrical, with more cancer lesion on the left than on the right. The PRPN of LPZ is relatively higher. LTZ has higher PRCF, and most of them were large lesions with high Gleason score $(\geq 8)$.

\section{Abbreviations, Disclosures, And Other}

\section{Abbreviations}

adjuvant Hormonal Therapy (AHT); cancer focus volume ratio (CFVR); confidence interval (CI); false negative rate (FNR); free prostate specific antigen ( $f-P S A)$; free/total prostate specific antigen ( $f / t P S A)$; left; peripheral zone (LPZ); left transitional zone (LTZ); left zone (LZ); odds ratio (OR); peripheral zone (PZ); positive rate of cancer focus (PRCF); positive rate of puncture needle (PRPN); prostate cancer (Pca); prostate volume (PV); PSA density (PSAD); radical prostatectomy (RP); right peripheral zone (RPZ); right transitional zone (RTZ); 
right zone (RZ); total prostate specific antigen (t-PSA); transitional zone (TZ); transperineal prostate biopsy (TPBx); transrectal prostate biopsy (TRBX)

\section{Acknowledgements}

I acknowledge all of the people who have contributed to this paper. Thanks to Ahmed Mahfouz for correcting this article. Additionally, my express my deepest gratitude foremost to Professor Zai-Sheng Zhu, my supervisor, for his constant encouragement and guidance. He has walked me through all the stages of writing this thesis. Without his consistent and illuminating instruction, this thesis could not have reached its present form.

\section{Availability of data and materials}

Reasonable requests for data and materials will be considered and should be

made in writing to the corresponding author.

\section{Authors' contributions}

Jia-Jun, Chen and Zai-Sheng, Zhu were involved in the manuscript conception and preparation. Jia-Jun, Chen; All authors collected and analyzed the data; Jia-Jun, Chen and Yi-Yi, Zhu carried out the statistical analysis; Jia-Jun, Chen and Zai-Sheng, Zhu performed the manuscript writing; All authors had final approval of the submitted version.

\section{Authors' Informations}

1 Jinhua Municipal Central Hospital, Department of Urology, No. 365 Renmin East Road, Jinhua City, Zhejiang Province, China 321000. ${ }^{2}$ Zhejiang University School of Medicine, No. 866 yuhangtang road, HangZhou, Zhejiang Province, China. ${ }^{3}$ Jinhua Municipal Central Hospital, Department of Ultrasound, No. 365 Renmin East Road, Jinhua City, Zhejiang Province, China 321000. ${ }^{4}$ Jinhua Municipal Central Hospital, Department of Pathology, No. 365 Renmin East Road, Jinhua City, Zhejiang Province, China 321000.

\section{Consent to publication}

Not applicable.

\section{Competing interests}

The authors declare that they have no competing interests.

\section{Ethics approval and consent to participate}

The study was approved by the medical ethics committee of Jinhua Central Hospital. All participants provided written consent.

\section{Funding}


Natural Science Foundation of Zhejiang Province. CN (Zhejiang Basic Public Welfare Research Project, No. LGF8H050006)

\section{Reference}

1. Bray F, Ferlay J, Soerjomataram I,et al. Global cancer statistics 2018: GLOBOCAN estimates of incidence and mortality worldwide for 36 cancers in 185 countries. [J/OL]. CA Cancer J Clin, 2018,68:394-424.

2. Ye Dingwei, Zhu Yao. Epidemiology of prostate cancer in China: an overview and clinical implication[J].

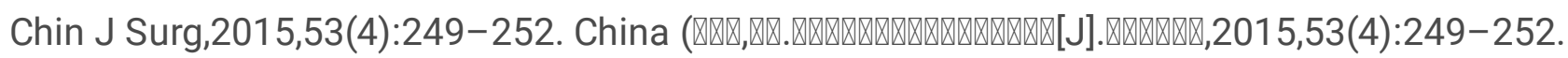
DOI:10.3760/cma.j.issn.0529-5815.2015.04.003.)

3. Zhou Liqun. Interpretation of consensus among experts on drug castration therapy for prostate cancer in China [J]. Chin J Urol ,2017,38(z1):4-5. China ( DOI:10.3760/j.issn.1000-6702.2017.z1.002.)

4. Hodge KK, McNeal JE, Terris MK, et al. Random systematic versus directed ultrasound guided transrectal core biopsies of the prostate [J]. J Urol,1989,142;71-75.

5. Chinese urology society, Chinese prostate cancer alliance. Consensus of Chinese experts on prostate puncture [J]. Chinese journal of urology, 2016,37(4):241-244. DOI: 10.3760/cma.j.issn.10006702.2016.04.001. China

6. Chang DT, Challacombe B, Lawrentschuk N. Transperineal biopsy of the prostate-is this the future? [J], Nat Rev Urol,2013,10:690-702.

7. Chun FK, Epstein Jl, Ficarra V, et al. Optimizing performance and interpretation of prostate biopsy a critical analysis of the literature[J]. Eur Urol, 2010,58:851-864.

8. Rabbani F, Stroumbakis N, Kava BR, et al. Incidence and clinical significance of false-negative sextant prostate biopsies[J]. J Urol,1998,159:1247-1250.

9. Epstein JI, Walsh PC, Sauvageot J, et al. Use of repeat sextant and transition zone biopsies for assessing extent of prostate cancer[J]. J Urol, 1997,158:1886-1890.

10. Schröder FH, van den Bergh RC, Wolters T, et al. Eleven-year outcome of patients with prostate cancers diagnosed during screening after initial negative sextant biopsies. [J]. Eur Urol. 2010 ,57:256-266.

11. Mabjeesh NJ1, Lidawi G, Chen J, et al. High detection rate of significant prostate tumors in anterior zones using transperineal ultrasound-guided template saturation biopsy. [J]. BJU Int,2012,110:993-997.

12. Gershman B, Zietman AL, Feldman AS, et al. Transperineal template-guided prostate biopsy for patients with persistently elevated PSA and multiple prior negative biopsies. [J]. Urol Oncol. 2013.31:1093-1097.

13. Bittner N1, Merrick GS, Butler WM, et al. Incidence and pathological features of prostate cancer detected on transperineal template guided mapping biopsy after negative transrectal ultrasound guided biopsy. [J]. J Urol. 2013,190:509-514.

14. Acher P1, Dooldeniya M. Prostate biopsy: will transperineal replace transrectal? [J]. BJU Int. 2013,112:533-534.

15. Pepe P, Aragona F. Prostate biopsy: results and advantages of the transperineal approach-twenty-year experience of a single center. [J]. World J Urol. 2014,32:373-377. 
16. Symons JL1, Huo A, Yuen CL, et al. Outcomes of transperineal template-guided prostate biopsy in 409 patients. [J]. BJU Int. 2013,112:585-593.

17. Kuru TH, Roethke MC, Seidenader J, et al. Critical evaluation of magnetic resonance imaging targeted, transrectal ultrasound guided transperineal fusion biopsy for detection of prostate cancer. [J]. J Urol. 2013,190:1380-1386.

18. Nguyen CT, Yu C, Moussa A, et al. Performance of prostate cancer prevention trial risk calculator in a contemporary cohort screened for prostate cancer and diagnosed by extended prostate biopsy. [J]. J Urol,2010,183:529-533.

19. Thompson IM, Ankerst DP, Chi C, et al. Assessing prostate cancer risk: results from the Prostate Cancer Prevention Trial. [J]. J Natl Cancer Inst,2006,98:529-534.

20. Breslow N, Chan CW, Dhom G, et al. Latent carcinoma of prostate at autopsy in seven areas. The International Agency for Research on Cancer, Lyons, France. [J]. Int J Cancer. $1977,20: 680-688$.

21. Kabalin JN, McNeal JE, Price HM, et al. Unsuspected adenocarcinoma of the prostate in patients undergoing cystoprostatectomy for other causes: incidence, histology and morphometric observations. [J]. J Urol. 1989,141:1091-1094.

22. Epstein JI, Srigley J, Grignon D, et al. Recommendations for the reporting of prostate carcinoma [J], Hum Pathol,2007,38®9®:1305-1309

23. Zhao li. Gleason score of prostate cancer and its clinical significance [J]. Journal of clinical surgery,2012,20(2):87-88. China [24] Dall'Oglio MF,Crippa A,Paranhos M,et al. Pathological extension of prostate cancer as defined by Gleason score on biopsy [J]. Int Braz J Urol,2005,31(4):326-330.

24. Vickers AJ,Cronin AM,Roobol MJロet al $\square$ The relationship between prostate-specific antigen and prostate cancer risk:the Prostate Biopsy Collaborative Group[J]. Clin Cancer Res,2010,16:4374-4381.

25. Xu JロThe Xus chart for prostate biopsy: a visual presentation of the added value of biomarkers to prostate-specific antigen for estimating detection rates of prostate cancer[J]ロAsian $\mathrm{J}$ Androl, 2014,16:536-540区

26. Moch $\mathrm{H}$, Humphrey PA, Ulbright TM, et al. WHO classification of tumors of the urinary system and male genital organ [M]. Lyon: IARC Press, 2016.

\section{Tables}

Tables 1 through 7 were included as Excel files in the supplemental files section.

\section{Figures}


Figure 1. Flow chart of 810 patients with TPBx

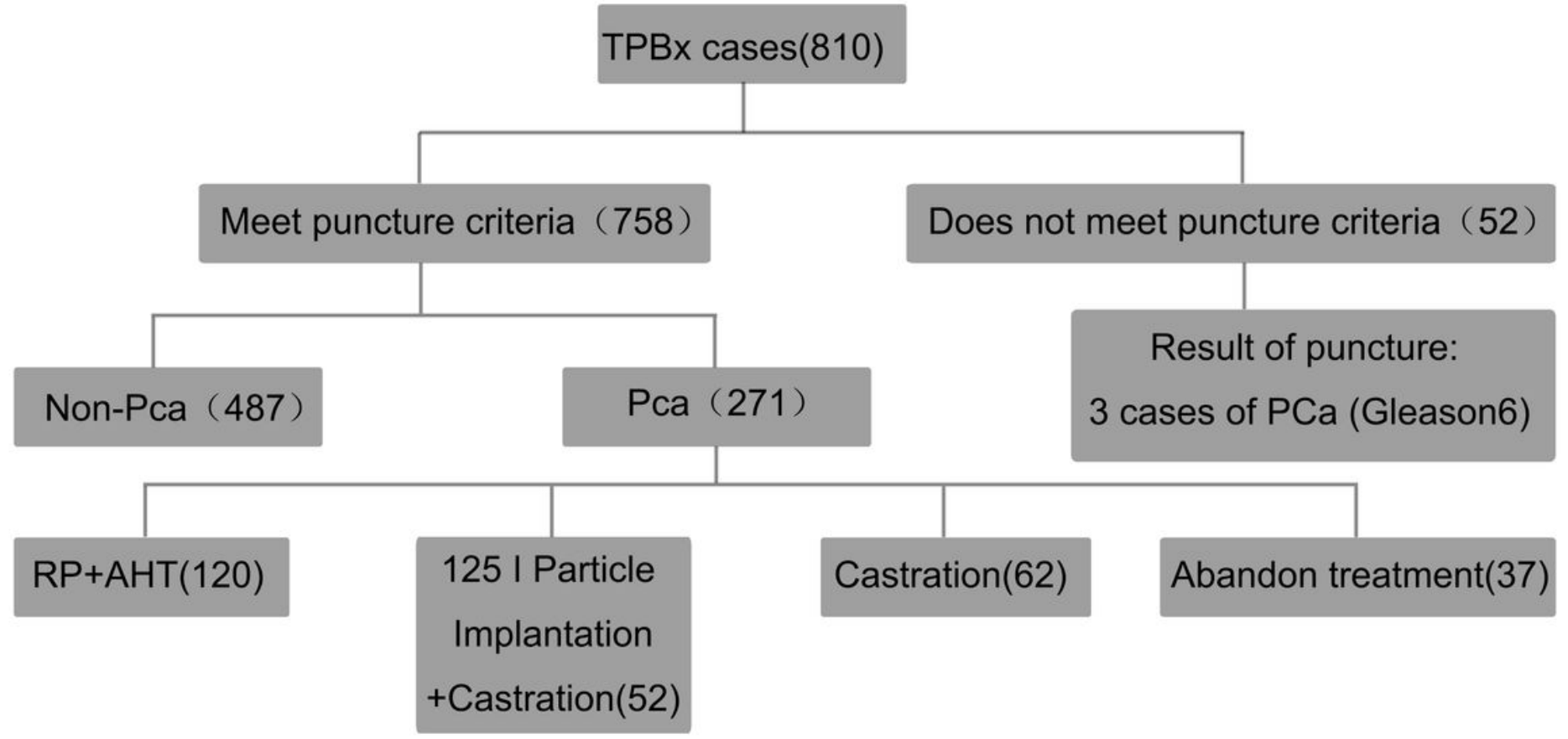

Figure 1

Flow chart of 810 patients with TPBx.

Figure 2. Example biopsy locations in a transperineal biopsy.

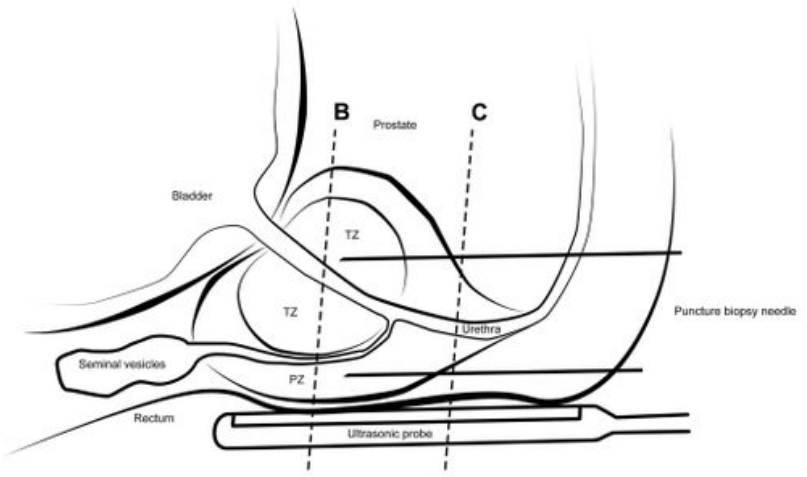

A.Schematic diagram of prostate sagittal plane

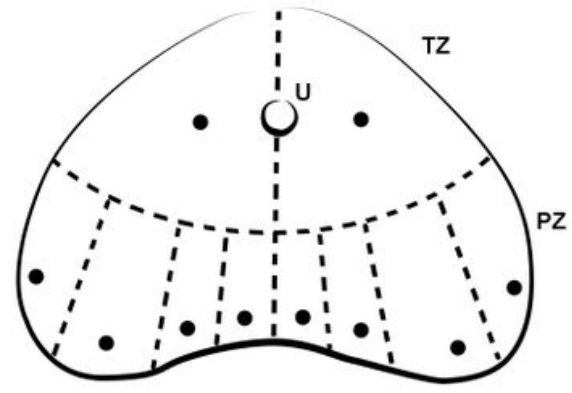

B. $10+X$

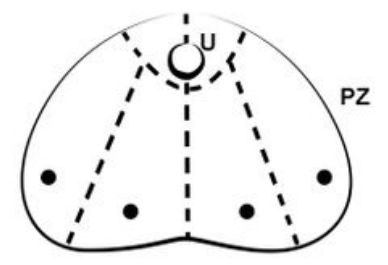

C.Apex of prostate

Figure 2

Example biopsy locations in a transperineal biopsy. 
Figure 3. ROC curves of age, t-PSA, PSAD, 1/PV, f-PSA, t-PSA/f-PSA in 758 TPBx patients

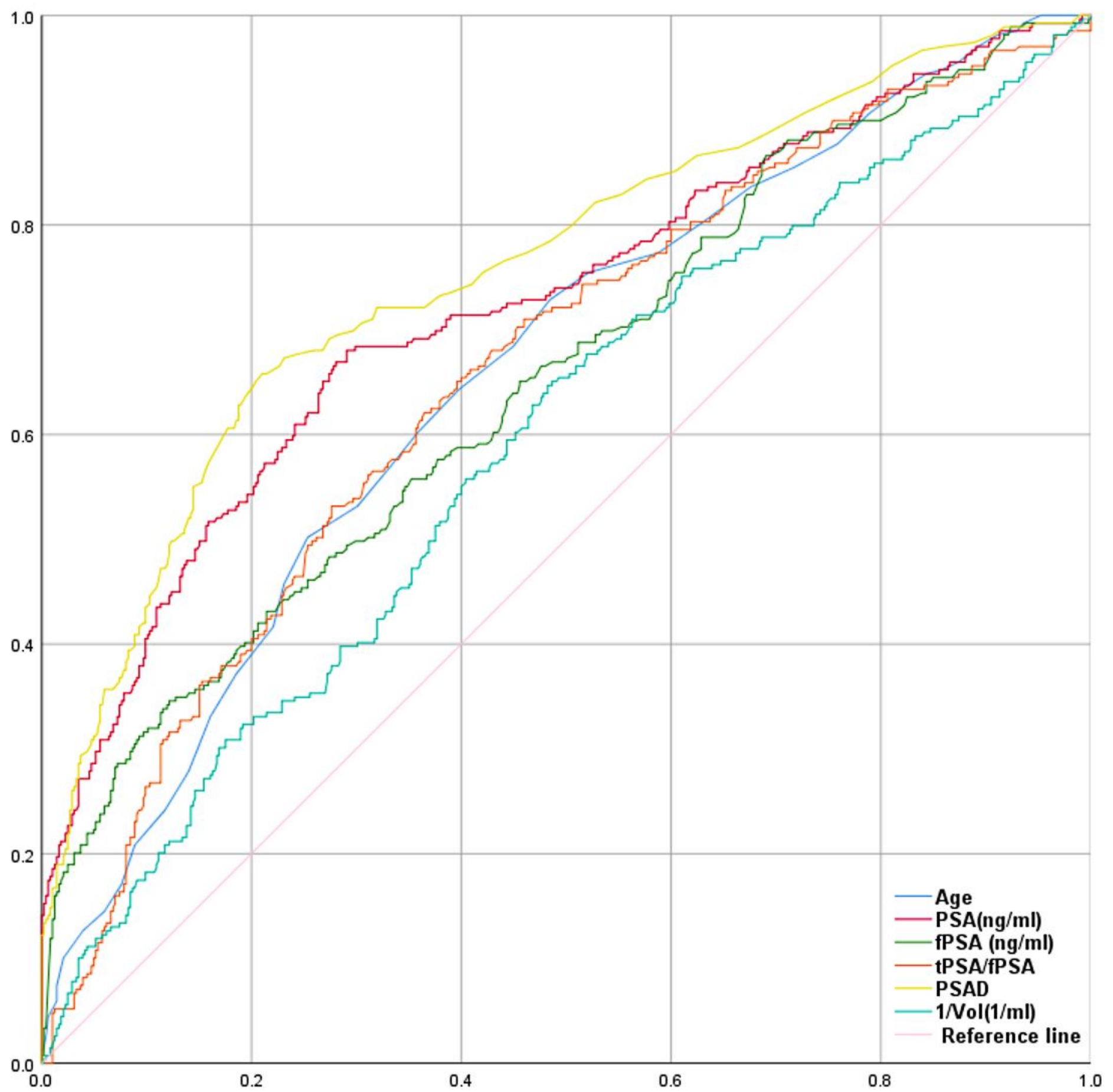

Figure 3

ROC curves of age, t-PSA, PSAD, 1/PV, f-PSA, t-PSA/f-PSA in 758 TPBx patients. 
Figure 4. Distribution of Gleason score in puncture biopsy of 271 patients

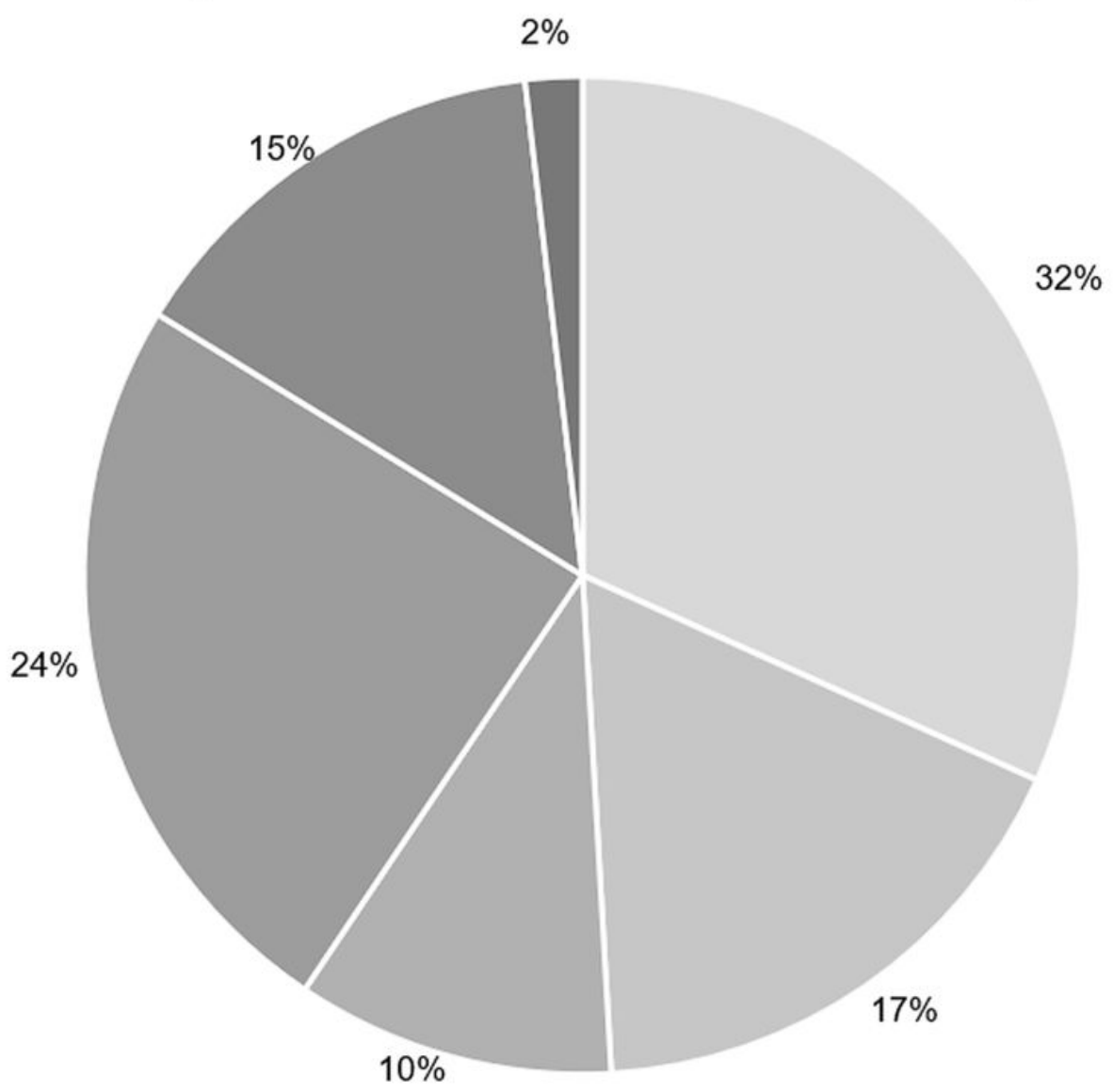

$$
\begin{array}{ll}
=6 & 86(32 \%) \\
=3+4 & 47(17 \%) \\
=4+3 & 28(10 \%) \\
=8 & 66(24 \%) \\
=9 & 39(15 \%) \\
=10 & 5(2 \%)
\end{array}
$$

Figure 4

Distribution of Gleason score in puncture biopsy of 271 patients.

\section{Supplementary Files}

This is a list of supplementary files associated with this preprint. Click to download.

- Table5.xlsx

- Table6.xlsx

- Table7.xlsx

- Table2.xlsx

- Table3.xlsx

- Table4.xlsx

- Table1.xlsx 